sequestrants were discontinued; loperamide was allowed as rescue therapy. Patients completed symptom diaries including stool frequency and Bristol Stool-form Scale (BSFS); a diarrhoea index ([stool frequency * mean BSFS] + loperamide use [weekly mg*3]) was calculated. Fasting serum FGF19 and total BA were measured before the first dose of OCA and after $2 \mathrm{w}$ treatment. Postprandial FGF19 and BA (6h area-under-curve, AUC) were determined after the first and last OCA dose. Data (expressed as medians) were analysed by Wilcoxon paired tests and Spearman correlation.

Results OCA increased fasting FGF19 from 133 to $237 \mathrm{pg} / \mathrm{ml}$ $(p=0.007)$ at $2 w$. Most patients had an increase $>60 \%$ in fasting FGF19 and a large OCA first dose/postprandial response. Fasting BA reduced from 1.5 to $0.9 \mu \mathrm{mol} / 1(p=0.13)$ and postprandial BA AUC was lower after the $2 \mathrm{w}$ OCA treatment (from 4.9 to $3.0 \mu \mathrm{mol} / \mathrm{l}$, $\mathrm{p}=0.02)$. Clinical improvements were found in all patients, including in stool frequency ( 23 to $14 / \mathrm{wk}, \mathrm{p}=0.02$ ), BSFS (5.15 to 4.34 , $p=0.05)$ and the diarrhoea index (113 to 76, $p=0.005)$. The reduction in BA AUC $(p=0.02)$ and the increase in fasting FGF19 $(p=0.03)$ both correlated with the reduction in stool frequency. Symptoms of abdominal pain, urgency and bloating also tended to be less on OCA treatment. OCA was well tolerated and no adverse events were reported of clinical concern.

Conclusion This study has shown for the first time that rational therapy with the FXR agonist OCA in PBAD is well tolerated and effective, stimulating serum FGF19 and reducing postprandial BA, resulting in clinical improvements in stool frequency and type. We propose larger, randomised, controlled trials of OCA. [EudraCT 2011-003777-28]

Disclosure of Interest None Declared.

\section{PTU-194 THE DIAGNOSTIC UTILITY OF COELIAC SEROLOGY IN LYMPHOCYTIC DUODENOSIS}

doi:10.1136/gutjnl-2013-304907.284

1," | Aziz, 'D M Smillie, 'D S Sanders. 'Department of Gastroenterology, Royal Hallamshire Hospital, Sheffield, UK

Introduction Lymphocytic duodenosis (LD) is defined by normal villous architecture and intraepithelial lymphocytes (IELs) $>25$ per 100 enterocytes. Such patients should not be diagnosed with coeliac disease (CD), solely by histology, as recent studies have suggested other associations with LD. Coeliac serology (tissue transglutaminase [TTG] and/or endomysial antibodies [EMA]) may play a useful role although their diagnostic value in such settings is unknown.

Aims To provide diagnostic outcomes in our expanding cohort of LD patients whilst also assessing the clinical utility of coeliac serology.

Methods Two hundred patients with LD were investigated for CD and other known associations of $\mathrm{LD}$, by means of revisiting the patient's history and recent investigations including the initial coeliac serology, followed by a combination of gluten challenge, HLA typing, repeat duodenal biopsies, and exclusion of infection/inflammatory bowel disease.

In the absence of an alternative cause, a diagnosis of CD was based on the persistence or progression of LD on a gluten containing diet, the presence of HLA DQ2 or DQ8, and a clinical response to a gluten free diet.

Results 150 female, 50 male, mean age 49, SD 16, age range 17-83

An identifiable association was found in $70 \%$ of patients - with CD (20\%), NSAIDs (17\%) and H,pylori (16\%) accounting for the majority. In $30 \%$ no cause was found, although reassuringly $2 / 3 \mathrm{rd}$ normalised their histology. The role of coeliac serology in $\mathrm{LD}$ for diagnosing $\mathrm{CD}$ is shown in table 1.

Conclusion As a single test, EMA has a greater diagnostic accuracy than TTG when assessing patients with LD.

As a combination test, only the presence of both a positive EMA and a raised TTG has a $100 \%$ predictive value for CD.
Abstract PTU-194 Table 1 The diagnostic utility of coeliac serology in lymphocytic duodenosis

\begin{tabular}{|c|c|c|c|c|}
\hline $\begin{array}{c}\text { LD coeliac serology test result } \\
(\mathrm{n} 200)\end{array}$ & PPV & NPV & Sensitivity & Specificity \\
\hline If only TTG performed & $54 \%$ & $92 \%$ & $70 \%$ & $85 \%$ \\
\hline If only EMA performed & $95 \%$ & $89 \%$ & $50 \%$ & $99 \%$ \\
\hline Both TTG and EMA normal & $6 \%$ & $44 \%$ & $20 \%$ & $16 \%$ \\
\hline TTG raised but EMA normal & $33 \%$ & $83 \%$ & $30 \%$ & $85 \%$ \\
\hline TTG normal but EMA positive & $80 \%$ & $82 \%$ & $10 \%$ & $99 \%$ \\
\hline Both TTG and EMA raised & $100 \%$ & $87 \%$ & $40 \%$ & $100 \%$ \\
\hline
\end{tabular}

Therefore, although coeliac serology is useful in $\mathrm{LD}$, most cases still require further work-up for diagnostic confirmation.

Disclosure of Interest None Declared.

\section{PTU-195 THE POPULATION PREVALENCE OF GLUTEN SENSITIVITY AND THE DIAGNOSTIC YIELD IN SECONDARY GASTROINTESTINAL CARE}

doi:10.1136/gutjnl-2013-304907.285

1."| Aziz, ${ }^{2} \mathrm{M}$ Hadjivassiliou, 'S Winfield, 'N Rugg, 'A Kelsall, 'L Newrick, 'D S Sanders. 'Gastroenterology; ${ }^{2}$ Neurology, Royal Hallamshire Hospital, Sheffield, UK

Introduction Healthcare professionals commonly encounter patients complaining of gluten sensitivity (GS) in the absence of serological and histological markers for coeliac disease (CD). This clinical entity has recently been termed non-coeliac gluten sensitivity (NCGS). The aim of this study was to determine the population prevalence of GS and to ascertain the diagnostic yield in those patients referred to secondary gastrointestinal (GI) care with gluten related symptoms.

Methods A population survey was conducted during March 2012 in Sheffield, UK, comprising basic demographic information, screening for GI conditions and enquiring for GS. We also analysed diagnostic outcomes in all patients referred by GPs to a dedicated secondary care clinic (2006-2012). The referral criteria were "GI symptoms attributed to gluten ingestion." Investigations included coeliac serology (EMA \& TTG), immunoglobulins, HLA DQ2/DQ8 typing, duodenal biopsies +/- gluten challenge if indicated. A diagnosis of CD was based on a positive coeliac serology, HLA typing and histological changes according to the Marsh classification.

Results 1002 adults completed the population based survey $(55 \%$ female, age range 16-93, mean age 39 yrs).

The prevalence of GS was 13\% (129/1002, female 80\% [P < 0.0001 ], age range $18-75$, mean age $39 \mathrm{yrs})$. The proportion of GS individuals who had seen a doctor for their symptoms was $35 / 129$ $(27 \%)$. In the absence of any known organic GI disease the prevalence of individuals fulfilling the ROME III criteria for IBS in the general population was $6 \%$, with up to $80 \%$ being female $(p<0.0001)$. Patients with IBS were more likely to report GS than non-IBS patients (43\% vs. $10 \%, \mathrm{p}<0.0001)$. GS individuals described a combination of intestinal \& extra-intestinal symptoms (Table 1). Of the GS cohort, 29\% (37/129) had tried a gluten free diet (GFD) - significant factors present in those trying a GFD include longer duration of symptoms (mean 96 vs. 54 months, $p=0.013$ ), previous doctor consultations (OR 52), diarrhoea (OR 17) and abdominal pain (OR 10.3).

In secondary GI care 156 patients with GS were investigated (85\% female, mean age $39 \mathrm{yrs}$ ). A diagnosis of CD was reached in $10 \%$ with the remaining being classified as NCGS. All patients with CD were HLA positive compared to $46 \%$ of NCGS cases. 
Abstract PTU-195 Table 1 Gluten sensitive symptoms reported in the adult community

\begin{tabular}{|ll|}
\hline Intestinal & Extra-intestinal \\
\hline Bloating 78\% & Fatigue 23\% \\
Abdominal discomfort 38\% & Headaches 22\% \\
Abdominal pain 29\% & Joint pains $8 \%$ \\
Constipation $21 \%$ & Rash $6 \%$ \\
Diarrhoea 16\% & Numbness $6 \%$ \\
Flatulence $14 \%$ & Confusion $5 \%$ \\
Nausea $9 \%$ & \\
\hline
\end{tabular}

Conclusion $13 \%$ of the general population self report GS. Of those investigated in the gastroenterology department only $10 \%$ have CD, with the remaining fulfilling the criteria for NCGS. The symptoms of NCGS are diverse and there is a relationship with IBS. The prevalence of a positive HLA typing in NCGS is half that of CD suggesting an alternative immune mechanism to gluten intolerance.

Disclosure of Interest None Declared.

\section{PTU-196 SMALL BOWEL CAPSULE ENDOSCOPY - A RETROSPECTIVE ANALYSIS OF 348 CASES FROM OEII AND LISTER HOSPITALS}

doi:10.1136/gutjnl-2013-304907.286

1."J A Evans, 'S Beg, 'R Nathwani, 'K Samsheer, 'E Ebro, 'E Turnbull. 'Department of Gastroenterology, Queen Elizabeth II \& Lister Hospitals, East \& North Herts NHS Trust, UK

Introduction Small bowel capsule endoscopy (SBCE) is a useful diagnostic tool for investigating iron deficiency anaemia (IDA) and suspected Crohn's disease (CD) ${ }^{1,2}$, but its use is often limited to specialist teaching centres. We aim to establish whether SBCE is a useful tool when employed in a district general hospital (DGH) setting.

Methods We retrospectively analysed the data of all SBCE carried out at QEII and Lister hospitals over 36 months since its introduction in 2009 using the GIVEN Pillcam SB 2 system. Patient demographics, indications, previous investigations, SBCE findings and how these altered management were reviewed.

Results 348 studies were performed on 175 males and 173 females; the mean age was 54.3 years (range 17-86). 207/348 (59\%) revealed some form of pathology. 7 studies were incomplete (2.01\%). Patency capsules were used in those with suspected risk of stricturing pathology for $22 / 348$ cases $(6.32 \%)$. No cases required surgical retrieval of a capsule.

The most common indication for SBCE was IDA = 185/348 studies $(53.1 \%)$. The next most common indication was suspected $\mathrm{CD}=141 / 348(40.5 \%)$. All patients had undergone bi-directional endoscopy prior to SBCE.

The commonest findings were; small bowel (SB) erosions/ ulcers (65 cases) SB angiodysplasia (39 cases) CD (21 cases) SB ulcers (19) polypoidal masses (13 cases) and fresh blood (11 cases). 3 cases of lymphoma and one of Enterobius vermicularis were discovered.

Where IDA was the indication, 65/185 studies (35.1\%) had findings that led to an alteration in management. In studies for suspected CD, 44/141 (31.2\%) had findings that led to an alteration in management.

Conclusion For IDA the diagnostic yield of 35\% is in keeping with previously published data, which shows that SBCE can identify a source of bleeding in $31-76 \% 1$.

For $\mathrm{CD}$ we identified abnormal pathology requiring further intervention in $31 \%$ of cases. It is debatable whether a small number of terminal ileal ulcers is sufficient to diagnose CD. If greater than 3 are seen, histological confirmation of $\mathrm{CD}$ is attempted via repeat endoscopy.

9 of the 348 patients had previously undergone a SBCE and the less invasive nature of SBCE lends itself to repeated use for small bowel CD surveillance.

SBCE is a reliable, safe and useful tool in the setting of a DGH setting for identifying small bowel pathology and should not be restricted to specialist centres.

Disclosure of Interest None Declared.

\section{REFERENCES}

R Sidhu, D Sanders, A Morris, M McAlindon. Guidelines on small bowel enteroscopy and capsule endoscopy in adults. BSG Guidelines. Gut 2008; 57:125-136.

SK Patel, P Mairs. A Review of Small Bowel Capsule Endoscopy Performed At Darent Valley Hospital Since Its Introduction in 2006. The Online Journal of Clinical Audits. 2011; Vol 3(3)

\section{PTU-197 A PATIENT SATISFACTION SURVEY FOR COELIAC DISEASE IN LEEDS - WHAT DO PATIENTS WANT?}

doi:10.1136/gutjnl-2013-304907.287

1."J S R Jennings. 'Gastroenterology, Leeds Teaching Hospitals NHS Trust, Leeds, UK

Introduction The long term follow up for patients with Coeliac disease is controversial. There are a number of models used in the UK which include follow up by primary care, conventional gastroenterology clinics, specialist Coeliac clinics \& dietician- or nurse-led services. National guidelines do not directly address this issue. I developed a patient satisfaction questionnaire with the Leeds local group of Coeliac UK to survey opinion.

Methods A 30 point patient directed questionnaire was sent electronically to members of the Leeds Coeliac UK group. The questionnaire asked for basic demographics, details of diagnosis, compliance with the gluten free diet (GFD), opinions on the service provided \& asked for a preference for the long term follow up.

Results 630 patients were sent an email copy of the questionnaire. I received 137 completed replies (22\%). Mean age 61 years (range 8-91), 78\% female, 96\% British Caucasian.

$18 \%$ had been diagnosed by their GP, 37\% a non-specified consultant, 35\% Gastroenterologist, 5\% colorectal surgeon, $6 \%$ Paediatrics \& $3 \%$ self diagnosed. Mean age at diagnosis was 48 (range 0.5 to 78 ) with a mean of 6 years from onset of symptoms to diagnosis.

$77 \%$ claimed to be strict with the GFD, 21\% described lapses with $2 \%$ variably compliant. $65 \%$ did not eat oats.

The majority (77\%) wished to be monitored long term. Currently $45 \%$ saw their GP only, 29\% attend general Gastroenterology clinics \& 26\% a Specialist Coeliac clinic. $82 \%$ described clinics as useful. In the future $28 \%$ preferred follow up to be provided by a GP with guidelines, $5 \%$ a general clinic, $60 \%$ a Coeliac clinic \& $5 \%$ a dietician-led service. $2 \%$ wanted no follow up. $68 \%$ wished to be seen yearly, $10 \% 2$ yearly.

Patients described their experience of in-patient care episodes as poor. $68 \%$ described staff knowledge of the GFD as average or poor, $87 \%$ had average or poor access to a GFD \& $73 \%$ described poor access to dietetic services on the ward. $83 \%$ described cafe or canteen services for a GFD as poor.

$59 \%$ had not received Pnemovax with $4 \%$ unsure.

Conclusion This is a large patient survey. The responders are clearly a self-selecting group. Even so a third prefer long term follow up with their GP, with the majority asking for specialist care. The poor number of Pneumovax recipients suggests guidelines are needed for primary care. This data may help guide the future development of services. Perhaps the time has come for a national survey. The experience of patients with Coeliac disease in hospital is a concern.

Disclosure of Interest None Declared 